J. Sustain. Wireless Syst.

Vol.02/ No. 2

Pages: 76-83

http://irojournals.com/irosws/

DOI: https://doi.org/10.36548/jsws.2020.2.003

\title{
An Optimized Packet Transfer through Wireless Network Using Age of Information
}

\author{
Dr. Joy Iong Zong Chen, \\ Professor, \\ Department of Electrical Engineering, \\ Da-Yeh University,Taiwan. \\ Email id: jchen@mail.dyu.edu.tw
}

\begin{abstract}
The cost for sampling a packet from transmission and transmitting the updates of the status using the wireless channel changes depending on the Age of Information (AoI) constraints. If any error occurs during the process of transmission, the decision lies with the scheduling algorithm to either retransmit the packet or to sample the packet again. Either way, the cost will include both transmission as well as sampling costs. However the aspect of sampling a new packet because of the error will result in increasing the cost of the system. With time average AoI constraint, we have devised a novel optimization technique that uses time average cost. In order to tackle this issue, we propose a decision making algorithm that decides in slots. The proposed algorithm arrives at one of the three solutions based on optimization technique. Accordingly, the user can either remain silent or transmit the old pack or sample the new packet. With the use of simulation results, the optimum performance of the proposed work in terms of AoI and timer average cost is observed and recorded.
\end{abstract}

Keywords: Age of Information; sampling; transmitting; Scheduling algorithm;

\section{Introduction}

The Age of information (AoI) is a novel technique introduced in the recent years to captivate the freshness and timeliness of the data gathered [1]. In was first identified in [2] wherein a definition for AoI has been given as the time elapsed since the last status update that was received at the receiving end. AoI is commonly used nowadays in a number of applications such as sensor networks, smart agriculture, environment monitoring etc. For example, let us take a cyber physical stream that is fit with many sensor samples and can send data such as solar radiation level, humidity and temperature which are considered to be data of freshness sensitivity. This data is transmitted via a wireless channel to the destination. An ideal destination which is in ideal condition will be receiving a continuous data flow of fresh data. But since nature intervenes, and because of the limited resources that are available, this is not a practical approach. Under these circumstances, it is important to maintain the data fresh and to manage the efficiency of the available resources. Hence, we take into account the transmission cost as well as the sampling of packets to propose an optimal algorithm which has minimal complexity that has the capacity to decrease the system's time average cost while ensuring that the average time of AoI is inside the threshold region. 
J. Sustain. Wireless Syst.

Vol.02/ No. 2

Pages: 76-83

http://irojournals.com/irosws/

DOI: https://doi.org/10.36548/jsws.2020.2.003

In the recent years, the performance of AoI in queuing system has gained a lot of importance. Moreover, in [3] Kam et al. have considered a mechanism which generates and transmits the packets in a random fashion through the network using dynamic routs to the remote monitor. He recommends the approach to find the analytical status age with the help of multiple queueing models such as $\mathrm{N}=\mathrm{N}=1, \mathrm{~N}=\mathrm{N}=2$, $\mathrm{N}=\mathrm{N}=3 \mathrm{etc}$. Similarly, in [4] the concept of cost of delay update and the importance of information was introduced by Kosta et al. They have also proposed a queue management system in [5] which gives the mathematical findings result for peakage as well as age information in $\mathrm{N}=\mathrm{N}=1$ system. AoI forms an integral part in recent advanced applications like energy harvesting capabilities of Internet of Things. In the research work by Kadota [6] and [7], different network scenarios are utilized for the age minimization problem. In [6] users that sample fresh information and further transmit it to the receiving end are considered for the proposed AoI minimization problem, through wireless channel. Likewise, in [7], the authors address the issue of random packet arrival at the destination for the same minimization of AoI problem. Initially, separately the packets are queued so that they hold fresh information which are accurately transmitted to the right destination. In [8], the authors use Markov Decision process for age these type of problems, giving the optimal off and in-line algorithms to solve the problem at hand. Similarly, in [9] a similar average AoI constraint on a wireless network with power minimization issue is considered. Here, an optimization theory known as Lyapunov optimization is introduced and its tools are utilized to formulate an algorithm that simultaneously maintains data fresh while decreasing the power consumption. In [10] He et. al have proposed a wireless network which is AoI constrained to solve energy minimization with link activation. However, there are very limited work in wireless network that takes into consideration both sampling as well as transmission costs using constraint of AoI in time average [11]. Hence, in order to solve this problem, we provide an optimized cost minimization solution for this system. Because of failure considerations, there are some doubts arise whether to attempt re-transmission of old packet or sample the new packet [12]. To solve this issue, under AoI constraint, we provide an optimized method of decreasing time average cost [13]. Using the tools of Lyapunov optimization theory, it is possible to find the solution and to develop an optimum algorithm with less complexity [14].

\section{Optimization Technique Proposed}

Let us consider $\mathrm{N}$ to be a new set of users that provide fresh sample information. This information is sent over the wireless fading channel as packets. If time is measured with slots such that $t \in Z_{+}$which is the $\mathrm{i}^{\text {th }}$ slot. At each time slot, the transmission scheduling and sampling are decided by the scheduler. During each slot, either of three decisions are taken, depending on the user. The user can decide to either remain silent or to transmit the previously sampled packet again or to sample fresh information and then transmit it. This method of decision making is denoted by $s_{i}(t)$. It can be represented as

$$
s_{j}(t)=\left\{\begin{array}{l}
1, \text { if user decides to sample } \\
0, \text { otherwise }
\end{array}\right.
$$


J. Sustain. Wireless Syst.

Vol.02/ No. 2

Pages: 76-83

http://irojournals.com/irosws/

DOI: https://doi.org/10.36548/jsws.2020.2.003

$$
N=\{1,2,3 \ldots \ldots, \quad N\}
$$

It should be noted that by the end of the slot, the information is sampled and based on the decision making algorithm transmits the information as packets to the end user. The decision of the user to sample the packet in t time slot can be represented as $\mu_{j}(t)$.

$$
\mu_{j}(t)=\left\{\begin{array}{l}
1, \text { if user } j \text { decides to transmit } \\
0, \text { otherwise }
\end{array}\right.
$$

In the $\mathrm{t}^{\text {th }}$ slot, if the user ' $\mathrm{j}$ ' begins to sample new information, then the transmission is attempted again inside the same slot. Hence $\mu_{j}(t)=1$ if $s_{j}(t)=1$. But if the user $\mathrm{j}$ retains the old sample without sampling the new information, then $\mu_{j}(t)$ can consider either the value 1 or 0 . The transmission takes place with fixed power. However, error during transmission is possible if fading channels are considered. The probability is $p_{i}^{1}$ when the packet is transmitted successfully to the receiver. However this probability is fixed for a period of time and will vary from one user to the other. We assume that on receiving the packet, the receiver will automatically acknowledge the positive reception of the packet by sending the signal ACK. Based on the constaint stated below, in one slot, one user can transmit the packet.

$$
\sum_{j=1}^{N} \mu_{j}(t) \leq 1, a t t
$$

At the receiver side, up to 1 packet can be received at every time slot. This can be denoted by

$$
d_{j}(t)=\left\{\begin{array}{l}
1, \text { if the packet is successfully transmitted by user } j \\
0, \text { otherwise }
\end{array}\right.
$$

The proposed work provides a low-complexity scheduling algorithm which makes use of the average age constraint in order to give an appropriate solution which is close to 1. Based on [10], the technique used is applied followed by [11] and [12] so that the constrains of time average age is also satisfied. A virtual queue is used to map the inequality constraint.

Let the virtual queue related to the constraint be represented by $\{\mathrm{Xi}(\mathrm{t})\} \mathrm{i} \in \mathrm{Nj}$. Every virtual queue is updated such that 
J. Sustain. Wireless Syst.

Vol.02/ No. 2

Pages: 76-83

http://irojournals.com/irosws/

DOI: https://doi.org/10.36548/jsws.2020.2.003

$$
X_{i}(t+1)=\max \left[X_{i}(t)-A_{i}^{\max }, 0\right]+A_{i}(t+1)
$$

Here,

$A_{i}^{\max }$ is the service rate, $A_{i}(t)$ is the arrivals and $X_{i}(t)$ is the queue

In order to further analyse the process, it is essential to understand the stochastic processes general theory. Let us take a system which has $\mathrm{K}$ queues. qi(t) and $q(t)=\left\{q_{k}(t)\right\}_{k \in K}$. Similarly, Let $L(q(t)$ and $\Delta(L(q(t))) \cong E\{L(q(t+1)-L(q(t+1)-L(q(t))) \mid q(t)$ represent Lyapunov drift and Lyapunov function respectively.

\subsection{Lypanov Function:}

A Lypanov Function can be represented as L: $R^{k} \rightarrow R$ and is said to have the below-said properties.

1. $L(x) \rightarrow+\infty$, as ||$x|| \rightarrow+\infty$

2. $L(x) \geq 0, \forall x \in R^{k}$

3. For the arguments, it is non - decreasing

\subsection{Lyapunov Drift}

When B is assigned positive values which belong to $\Delta(L(q(t))) \leq B-\sum_{k=1}^{k} q_{n}(t)$ for the different time slots taking into account the stability of $q(t)$.

\subsection{Optimization Problem}

The stochastic optimization problem can be defined based on the time average costs expected along with AoI definition

$$
\min _{s(t), \mu(t)} \bar{c}
$$


J. Sustain. Wireless Syst.

Vol.02/ No. 2

Pages: 76-83

http://irojournals.com/irosws/

DOI: https://doi.org/10.36548/jsws.2020.2.003

$$
\begin{aligned}
& \sum_{i=1}^{K} \mu_{i}(t) \leq 1, \forall t \\
& \bar{A}_{\imath} \leq A_{i}^{\max }, \forall i \in N \\
& \mathrm{~s}(\mathrm{t}), \mu(\mathrm{t}) \in\{0,1\}^{N}
\end{aligned}
$$

where $\mathrm{s}(\mathrm{t})=\left[\mathrm{s}_{1}(\mathrm{t}), \ldots \ldots \ldots \ldots, \mathrm{s}_{\mathrm{N}}(\mathrm{t})\right.$ and $\mu(\mathrm{t})=\left[\mu_{1}(t), \ldots \ldots \ldots, \mu_{N}(t)\right.$. The maximum time average age constraints expected and interference are represented in the equation. The purpose of our work is to find the optimal solution which lies below the threshold in terms of average age and also decreases the system cost.

\subsection{Policy of Penalty}

In order to decrease the sum of penalty function and Lyapunov drift, a drift-plus-penalty is proposed. The DPP algorithm is applied such that while the virtual queues are stabilised, the time average costs are also minimized simultaneously. i.e. the upperbound of the following equation is to be minimized. $\Delta(X(t))+V E\left\{c(t) \mid S_{t}\right\}$ Where penalty is measure with the help of V which is the weight. Similarly the expression for an upper bound is represented in the equation below

$$
\begin{aligned}
& \Delta(X(t))+V E\left\{c(t) \mid S_{t}\right\} \\
& \quad \leq B+\sum_{i=1}^{N} E\left\{X_{i}(t)\left[A_{i}^{p}(t+1) W_{i}(t)+\left(A_{i}(t)+1\right)\left(1-W_{i}(t)\right)-A_{i}^{\text {max }}\right] \mid V E\left\{c(t) \mid S_{t}\right.\right.
\end{aligned}
$$

\section{Results}

This section analysis the results observed through simulation process in order to find the performance of our proposed work with respect to cost of the system and average AoI. We have considered an unreliable wireless channel to transmit packets (fresh data) from two users. Initially, we examine how cost and average AoI are affected by the importance factor V. Then the scheduler's behaviour is taken into consideration where the success probability of the two users vary. Further, the simulation results are taken for the different success probabilities and the effect on cost of the system as well as AoI time average. 
J. Sustain. Wireless Syst.

Vol.02/ No. 2

Pages: 76-83

http://irojournals.com/irosws/

DOI: https://doi.org/10.36548/jsws.2020.2.003

When $\mathrm{p} 1=0: 6$ and $\mathrm{p} 2=0: 9$, the results of the system are represented in Figs. 1 and 2. Fig. 1 . The figure Fig. 1 represents the average AoI of the two users for ' $\mathrm{V}$ ' which is the important factor. The factor ' $\mathrm{V}$ ' is generally multiplied with cost to arrive at the final number. Hence by increasing the value of $\mathrm{V}$, we decrease the cost of the system.

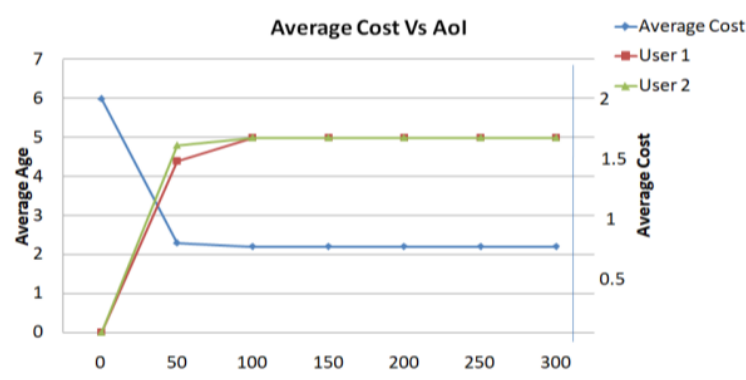

Fig.1.Average cost Vs Average AoI

Fig.2. indicates average number of samples vs average number of transmissions as shown. However as observed in Fig. 1. AoI is highly affected for high V value. It is observed that A1 takes the value of less than $A_{\max }$ when $V=50$ while at $V=300$, it takes the value $A_{\text {min. }}$. Moreover, Fig.1. portrays that since the probability of success is very low, the user 1 will have a higher average value for the values of $\mathrm{V}$ between 1 and 100. The results are also verified theoretically for the average AoI constraints. Based on the simulation results, it is also observed that there is an increase in average AoI as there is a decrease in success probability since it will require more time to complete a transmission successfully. Hence after a couple of attempts by the user to the receiver the pack will arrive, thereby increasing AoI. Similarly, Fig.3 portrays the average AoI for two users along with the cost consideration depending on $\mathrm{p}_{1}$ and $\mathrm{p}_{2}$ which are the success probabilities.

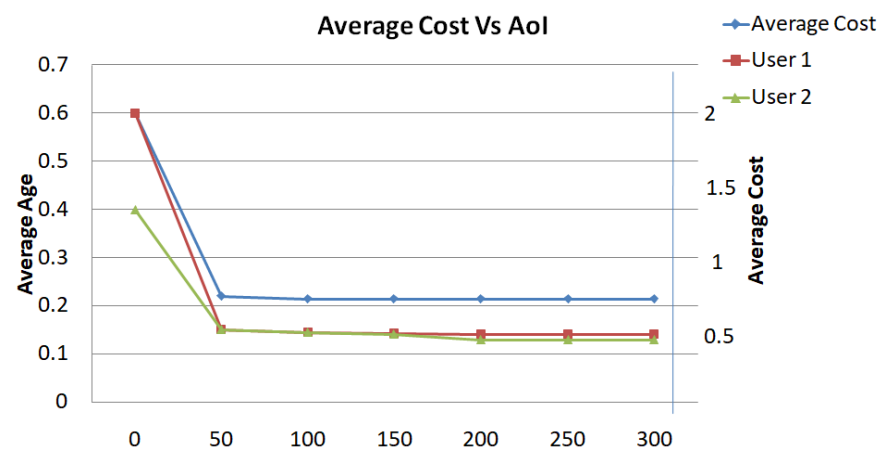

Fig.2. Average number of transmissions and average number of samplings 
J. Sustain. Wireless Syst.

Vol.02/ No. 2

Pages: 76-83

http://irojournals.com/irosws/

DOI: https://doi.org/10.36548/jsws.2020.2.003

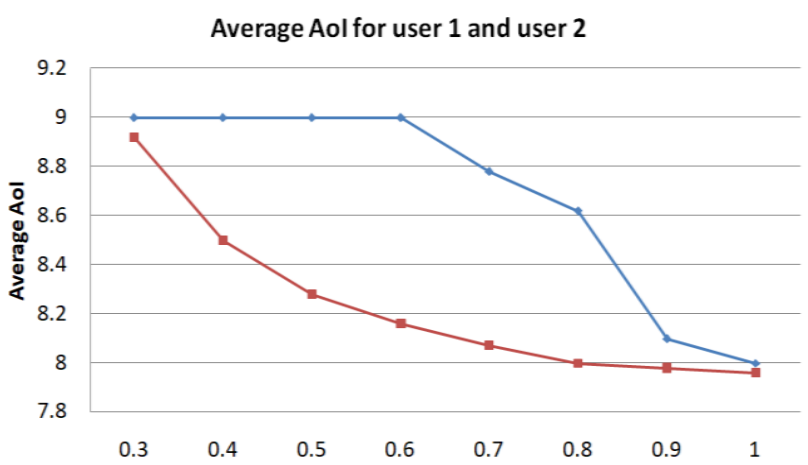

Fig.3. Average AoI

\section{Conclusion}

In the proposed algorithm, we have minimized the cost time average of the transmission over the wireless system using the algorithm which decides transmission scheduling and sampling during the time slots based on AoI constraint. Lyapunov optimization serves as the base for the proposed methodology. In the proposed work, it is possible to arrive at a solution that is nearer to the optimal solution. Both sampling as well as transmission cost are taken into consideration. Moreover, there is need for optimizing transmission as there is possibility of error occurrence due to fading. The proposed work is analysed through simulation results in order to find the performance of the system with respect to cost and AoI. Based on the results, it is observed that there is a trade-off between cost and AoI and the impact of success probabilities on the system's performance.

\section{References}

[1] A. Kosta, N. Pappas, and V. Angelakis, "Age of information: A new concept, metric, and tool," Foundations and TrendsR in Networking, vol. 12, no. 3, pp. 162-259, 2017.

[2] S. Kaul, R. Yates, and M. Gruteser, "Real-time status: How often should one update?," in Proc. IEEE INFOCOM, pp. 2731-2735, Mar. 2012.

[3] C. Kam, S. Kompella, G. D. Nguyen, and A. Ephremides, "Effect of message transmission path diversity on status age," IEEE Trans. Inf. Theory, vol. 62, no. 3, pp. 1360-1374, Mar. 2016.

[4] A. Kosta, N. Pappas, A. Ephremides, and V. Angelakis, "Age and value of information: Non-linear age case,” in. Proc. IEEE ISIT, pp. 326-330, June 2017.

[5] A. Kosta, N. Pappas, A. Ephremides, and V. Angelakis, "Age of information performance of multiaccess strategies with packet management," Journal of Commun. and Networks, vol. 21, no. 3, pp. 244-255, June 2019. 
J. Sustain. Wireless Syst.

Vol.02/ No. 2

Pages: 76-83

http://irojournals.com/irosws/

DOI: https://doi.org/10.36548/jsws.2020.2.003

[6] Kadota, A. Sinha, and E. Modiano, "Scheduling algorithms for optimizing age of information in wireless networks with throughput constraints," IEEE/ACM Trans. Net., vol. 27, no. 4, pp. 1359-1372, 2019.

[7] I. Kadota and E. Modiano, "Minimizing the age of information in wireless networks with stochastic arrivals," arXiv preprint arXiv:1905.07020, 2019.

[8] Y.-P. Hsu, E. Modiano, and L. Duan, "Age of information: Design and analysis of optimal scheduling algorithms,” in Proc. IEEE ISIT, pp. 561- 565, June 2017.

[9] M. Moltafet, M. Leinonen, M. Codreanu, and N. Pappas, "Power minimization in wireless sensor networks with constrained AoI using stochastic optimization," in Proc. Asilomar, Nov. 2019.

[10] L. Georgiadis, M. J. Neely, and L. Tassiulas, "Resource allocation and cross-layer control in wireless networks," Foundations and TrendsR in Networking, vol. 1, no. 1, pp. 1-144, 2006.

[11] Muneera Begum H, D. A. Janeera, and AG, Aneesh Kumar. "Internet of Things based Wild Animal Infringement Identification, Diversion and Alert System" In Fifth International Conference on Inventive Computation Technologies (ICICT-2020), pp. 672-676. IEEE, 2020.

[12] Haoxiang, W., \& Smys, S. (2020). Soft Computing Strategies for Optimized Route Selection in Wireless Sensor Network. Journal of Soft Computing Paradigm (JSCP), 2(01), $1-12$.

[13] Raj, J. S. (2020). Machine Learning Based Resourceful Clustering With Load Optimization for Wireless Sensor Networks. Journal of Ubiquitous Computing and Communication Technologies (UCCT), 2(01), 29-38.

[14] Ranganathan, G., \& Smys, S. Smart Wireless sensors for Impairment detection of the offshore Wind Turbines.

\section{Author's Biography}

Dr. Joy Iong-Zong Chen is currently a full professor of Department of Electrical Engineering Dayeh University at Changhua Taiwan. Prior to joining the Dayeh University, he worked at the Control Data Company (Taiwan) as a technical manager since Sep. 1985 toSep. 1996. His research interests include wireless communications, spread spectrum technical, OFDM systems, and wireless sensor networks. He has published a large number of SCI Journal papers in theissues addressed physical layer for wireless communication systems. Moreover, he also majors in developing some applications of the IOT (Internet of Thing) techniques and Dr. Joy I.-Z. Chen owned some patents authorized by the Taiwan Intellectual Property Office (TIPO) 